\title{
Accumbens D2: Raters of the Loss Outcome
}

\author{
Andrew T. Marshall $^{1}$ • Kimberly Kirkpatrick ${ }^{1}$
}

Published online: 21 July 2016

(C) Psychonomic Society, Inc. 2016

\begin{abstract}
Zalocusky et al. (Nature 531:642-646, 2016) recently showed that activity in D2R+ cells in the nucleus accumbens is associated with loss sensitivity to prior outcomes and reduced subsequent risky choice, and that optogenetic stimulation of these cells decreased risky choices in risk-prone rats. While their findings are important for understanding trait-level risktaking, future research should aim to examine the neuronal mechanisms of a broader range of facets of gain and loss processing with respect to different potential reference points.
\end{abstract}

Keywords Risky choice $\cdot$ Probability discounting $\cdot$ Loss sensitivity $\cdot$ Nucleus accumbens $\cdot$ Dopamine

Risky choice, or probability discounting, has received considerable attention recently, as it is a trait that predicts several other maladaptive behaviors. Risky choice involves choosing between a "safe" outcome (which is smaller, but certain to occur) versus a riskier outcome (which is larger, but less certain). Uncertainty is induced through the possibility of receiving a risky-choice outcome that is even smaller than the safe outcome (a "non-zero" loss) or through the possibility of receiving nothing (a "zero-valued" loss). Variants on the risky choice task have been developed for use in animals (particularly rats), providing an important avenue for examining the neural substrates of risky choice. This approach was used in a recent article by Zalocusky, Ramakrishnan, Lerner, Davidson, Knutson, and Deisseroth (2016).

Kimberly Kirkpatrick

kirkpatr@ksu.edu

1 Department of Psychological Sciences, Kansas State University, 492 Bluemont Hall, 1100 Mid-Campus Drive, Manhattan, KS 66506, USA
With regard to trait-level risk-taking, most individuals express a fairly consistent pattern of risk aversion, whereas some show a more problematic pattern of risk proneness. While stable individual differences have been documented in rats, the Zalocusky et al. paper is one of the first to attempt a comprehensive analysis of the neural substrates of trait risky choices in a rat model. One notable feature of their paper is the use of multiple converging neurobiological techniques to examine the neural substrates of trait risky choice. First, they used a pharmacological approach to identify the potential receptor subtypes critical for trait risky choice. They found that pramipexole (PPX), a D2/D3 agonist, increased risky choice, which has important implications for understanding the effects of D2/D3 agonists on increased risk-seeking in Parkinson's patients. Next, they demonstrated that PPX infusions into the nucleus accumbens (NAC), a key brain region within the reward system, increased risk preference, but that there was no effect of infusions of PPX into the orbitofrontal cortex, a region associated with a range of other decision-making functions. These results suggest that NAC dopamine cells affect trait risky choice, ultimately representing an important and novel contribution to the field, although the degree of selectivity of D2 involvement is still an open question.

Having identified NAC dopamine as a potentially key factor, Zalocusky et al. then conducted confirmatory analyses. First, they measured NAC D2R+ cells and found correlations of the timing and rate of neural activity with both recent losses and the upcoming choice. This suggests that these cells may be important for encoding losses and directing lose-shift behaviors, which result in a reduction in gambling following losses. Loss processing has been shown to be deficient in pathological gamblers, so identifying the key substrates for loss processing is an important step towards understanding the factors that may contribute to pathological gambling. Finally, the NAC D2R+ cells were subjected to optogenetic 
activation during the decision period, resulting in decreased risk-seeking behaviors in risk-prone individuals. The combined results suggest that NAC D2 cells play a key role in influencing overall trait risk-taking.

Given the critical nature of risky choices for many facets of human and animal behavior, understanding the multitude of neural and behavioral mechanisms that contribute to risky choice is an important venture. To that end, the Zalocusky et al. paper is a launching point for further work. However, the factors that contribute to risky choice are numerous, complex, and still poorly understood in many ways, so it seems that they have opened opportunities for future research. In the Zalocusky et al. paper, risky choice is measured using a "risk variability" procedure, in which uncertainty is induced by exposing the rats to non-zero losses (i.e., risky choices resulted in a larger or smaller amount of food compared to safe choices). Non-zero losses, in which a small amount of food is delivered, have different effects compared to zero-valued losses, in which reward is omitted entirely. Thus, as a starting point, it would be interesting to determine whether the NAC $\mathrm{D} 2 \mathrm{R}+$ cells are also critical for loss sensitivity with zerovalued losses, if zero-valued losses are processed by other NAC cells/receptor subtypes (e.g., D3), or if these types of losses are processed by a different region entirely. In addition, their research was conducted in a closed economy, in which the rats' entire water consumption occurred during the experiment. Open versus closed economies impact decisionmaking processes and can induce different motivational states, so it is possible that economy type could affect the expression of trait risk-taking. Similarly, closed economies may induce state-based increases in risk-taking that may cloud conclusions regarding general risk aversion/proneness in animals (see Caraco, 1981). Finally, the safe and risky options were matched in terms of expected value, so that there was no incentive to maximize reward. While this approach provides a means of measuring trait impulsivity in the absence of maximizing, real-world decisions rarely involve options that are perfectly matched in expected value. None of these issues undermine the Zalocusky et al. study, but they provide important qualifiers for the interpretation of their results: We can only conclude that NAC D2R+ cells contribute importantly to risky choice in closed economies with matched expected values, and are potentially important for loss sensitivity involving non-zero losses. Future work should aim to extend these findings to other risky choice approaches, using different parameters and paradigms.

A second key issue for future work relates to the subjective perception of both "gains" and "losses." Gains and losses can be judged relative to different reference points and reference-point use may be affected by the current motivational state of the animal (e.g., Wang, \& Johnson, 2012). Within the standard risky choice paradigm, a loss on the risky side could be gauged relative to the risky side "win" amount, the safe side amount, or, in the case of non-zero losses, could be judged relative to zero ("something is more than nothing"). Similarly, gains can be judged relative to different reference points. To complicate matters further, some losses can be disguised as wins, thus undermining their ability to effectively reduce risk-taking. Therefore, the perception of gains and/or losses is subject to both individual and contextual variation. This creates a challenge for understanding factors such as loss sensitivity, with which NAC $2 \mathrm{R}+$ cells were associated in the Zalocusky et al. paper. Overall, it is clear that future work needs to employ methods for parsing out different reference points by differentiating and identifying individual differences in gain and loss processing (see Seymour, Maruyama, \& De Martino, 2015). These different constructs may then be isolated to specific neural regions and circuits.

A final key issue relates to the neurobiological approaches that can be employed in future work. While NAC dopaminergic cells appear to participate in trait risky choice, it would be interesting to examine the inputs to those cells and the projections of those cells to understand the circuit-level functionality and the participation of direct and indirect dopamine pathways in the cognitive and behavioral issues discussed above. In addition, because PPX strongly activates D3 as well as D2, it would be valuable to examine the contribution of D3 to outcome processing and trait risky choice. Decision making is a dynamic process, and examining a wider range of neurobiological factors will be an important step for fully understanding the complexity of neuronal processes involved in trait risky choice.

This is an exciting time for studying the neurobiology of risky decision making. The field has developed sophisticated behavioral methods for parsing out different aspects of choice behavior, and there now are a range of modern neuroscience techniques that can be brought to bear in identifying the neuronal architecture and neural computations that underlie different facets of risky choice behavior. Zalocusky et al. have opened a door for the next stage of work in investigating this important problem.

\section{References}

Caraco, T. (1981). Energy budgets, risk and foraging preferences in darkeyed juncos (Junco hyemalis). Behavioral Ecology and Sociobiology, 8(3), 213-217.

Seymour, B., Maruyama, M., \& De Martino, B. (2015). When is a loss a loss? Excitatory and inhibitory processes in loss-related decision making. Current Opinion in Behavioral Sciences, 5, 122-127. doi:10.1016/j. cobeha.2015.09.003

Wang, X. T., \& Johnson, J. G. (2012). A tri-reference point theory of decision making under risk. Journal of Experimental Psychology: General, 141(4), 743-756. doi:10.1037/a0027415

Zalocusky, K. A., Ramakrishnan, C., Lerner, T. N., Davidson, T. J., Knutson, B., \& Deisseroth, K. (2016). Nucleus accumbens D2R cells signal prior outcomes and control risky decision-making. Nature, 531(7596), 642-646. 